\title{
Does culture condition of reduced oxygen pressure helps in embryo quality? A prospective randomized study
}

\author{
Deepak Patil*
}

Department of Obstetrics and Gynaecology, Command Hospital Air Force, Bangalore, Karnataka, India

Received: 04 May 2021

Accepted: 18 May 2021

\section{*Correspondence:}

Dr. Deepak Patil,

E-mail: deepakaarav6@gmail.com

Copyright: () the author(s), publisher and licensee Medip Academy. This is an open-access article distributed under the terms of the Creative Commons Attribution Non-Commercial License, which permits unrestricted non-commercial use, distribution, and reproduction in any medium, provided the original work is properly cited.

\begin{abstract}
Background: Embryo quality is of paramount importance in an in vitro fertilization and embryo transfer (IVF-ET) cycle. A healthy embryo leads to a robust pregnancy. Incubation in an ideal atmosphere is essential requirement in an IVF-ET cycle.

Methods: Traditionally carbon dioxide incubator with sensors for carbon dioxide levels and temperature has been used widely. We compared the quality of these embryos to incubation in hypoxic condition by addition of nitrogen. Oxygen levels were brought to $5 \%$ as compared to $21 \%$ in room air.

Results: Ladies with high body mass index (BMI) and advanced age had more embryos available for transfer. More embryos reached day 3 and day 5, thus increasing availability for cryopreservation. The fragmentation rate and fertilization failure were less. Pregnancy rate was definitely improved as compared to traditional incubation.

Conclusions: Hypoxic incubation condition leads to better embryo health. This translates into improved and efficient cycle outcome.
\end{abstract}

Keywords: Embryo culture, Fragmentation rate, Vitrification

\section{INTRODUCTION}

From initial days of embryo culture in jars by Edward and Steptoe, in vitro fertilization and embryo transfer (IVFET) has come a long way. Literally in-vitro means a glass jar in Latin. It was pioneering efforts of consultant gynecologist Patrick Steptoe and research scientist Robert Edwards which bought about the birth of Louis brown in 1978. Their work initially faced criticism due to fear of rupture in assumed relationship of human reproduction and nature. Finally with the birth of Louis Brown, it was celebrated as world first medical and media event. This event also attested to the safety and scientific breakthrough of their efforts. ${ }^{1}$ The health aspects and cost factor in an IVF-ET program has been researched and improved upon by various reproductive medicine teams. With personalized stimulation protocol incidence of ovarian hyperstimulation syndrome (OHSSS) has been reduced. The ovum pickup procedure with improvement in anesthetic drugs and surgical disposables has been made outpatient in most of the centres. With cryopreservation of supernumery embryos cumulative pregnancy rate has increased.

In vivo studies using flexible polarographic microelectrodes in rhesus monkeys have shown reduced oxygen tension in uterus and tubes as compared to ambient oxygen tension. ${ }^{2}$ This concept has been utilized to provide a reduced oxygen level in culture of gametes. Addition of nitrogen is used to displace oxygen to $5 \%$.The entire aim of embryology team is to provide a suitable and near in vivo environment to gametes.

Objective of our study was to assess the impact of reduced oxygen level on embryo quality by comparing to standard incubation in carbon dioxide incubator under room air oxygen tension. Also we assessed the impact of this on vitrification and pregnancy rate. 


\section{METHODS}

\section{Material and methods}

This multicentric prospective randomized study was done in a tertiary care assisted reproductive techniques centre of armed forces. Records were assessed to study the impact of culture condition of reduced oxygen pressure.

\section{Study design}

The design of the study was multicentric and prospective randomized study.

\section{Study location}

The study was conducted at a tertiary care ART centre.

\section{Study duration}

The duration of the study was from January 2019 to December 2019.

\section{Subjects and selection method}

100 couples going IVF ET meeting the inclusion and exclusion criteria were recruited. Randomization was provided by choosing alternate methods of incubation, traditional, environmental ( $20 \%$ oxygen) or physiological, hypoxic (5\% oxygen) in consecutive couples. 50 couples were selected in each arm of the study. All couples screened for IVF-ET program with corrected hematological and biochemical parameters were included. Couples with prior fertilization failure were not included in the study. Ladies with endometrioma and hydrosalphinx, detectable with pelvic ultrasound were excluded from the study.

Stimulation protocol for standardization only antagonist protocol for stimulation were recruited. Patients were started on injection recombinant follitropin alpha (Merck Pharmaceuticals) $1050 \mathrm{IU} / 1.75 \mathrm{ml}$ powder with solvent for stimulation from day 2 of menstrual cycle. Personalized stimulation protocols were started based on age, body mass index (BMI), ovarian volume, anti-mullerian hormone $(\mathrm{AMH})$ and previous stimulation data if any. Injection ovurelix containing citrorelix $0.25 \mathrm{mg}$ from Sun Pharmaceuticals was started as antagonist on evidence of sufficient endogenous estrogen production. On adequate stimulation with a cohort of at least 4 follicles of size 18 $\mathrm{mm}$, trigger was given with injection ovitrelle $250 \mathrm{mcg}$ (contains recombinant choriogonadotropin alpha, 250 mcg, Merck ltd) and ovum pickup was done after 36-40 hours.

\section{Ovum pick up (OPU) and IVF-ET}

The aspirated oocyte cumulus complexes (OCC) were double washed in 4-(2-hydroxyethyl)-1piperazineethanesulfonic acid (HEPES) based media.
They were dissected free of extra cumulus and blood clots under magnification and transferred to bicarbonate-based media in micro drop (50 micro liters) culture system with overlay of mineral oil.

All the media required for the OPU were aliquoted on the previous evening for overnight equilibration.

Heracell 150i $\mathrm{CO}_{2}$ incubator was used for incubation in $20 \%$ environmental oxygen condition. Appendorf Cellxpert C170i incubator with oxygen control option of $1-20 \%$ was used for hypoxic incubation. IVF ET grade nitrogen gas was used to reduce oxygen level to $5 \%$. Carbon dioxide level was kept at $6 \%$ and temperature set at 37 degree Celsius.

Semen preparation was done using double density gradient method. The liquefied semen sample was overlaid, over a double density gradient of ORIGIO gradient 40/80 from Cooper surgical. For swim up universal IVF medium from Cooper surgical was used.

Conventional insemination was done only if post wash specimen shows a sperm concentration of more than 20 million/ml with more than $50 \%$ grade 4 motility. Else ICSI was used. For culturing the embryos and insemination, micro drops of 50 micro liters of universal fertilization media under mineral oil were used. Five such micro drops were made in one $60 \mathrm{~mm}$ human IVF grade nunc petri dish. Each such micro drop contained not more than 4 oocytes.

Fertilization was assessed after 16-18 hours of insemination. Culture media was changed after every 24 hours of incubation with fresh plates. Cleavage and fragmentation was reassessed every 24 hours. Decision to continue growing the embryo, vitrify or transfer was based on numerous parameters. They included: number of oocytes retrieved, fertilization rate, quality of embryo, evidence of any infection, and patient consent.

Luteal support was provided with injection algest containing micronized progesterone $100 \mathrm{mg} / 2 \mathrm{ml}$ intramuscular (IM) per day along with tablet duphastone $10 \mathrm{mg}$ from Abott Pharmaceuticals containing dydrogesterone $10 \mathrm{mg}$ twice a day for 15 days.

Tablet progynova $2 \mathrm{mg}$ was added based on endometrial thickness. Beta human chorionic gonadotropin (HCG) was done on day 15 . Values above $5 \mathrm{miu} / \mathrm{ml}$ were used as positive for calculating pregnancy rate. Clinical pregnancy was defined as ultrasound detection of embryonic cardiac activity. Chi square test was used for data analysis.

\section{RESULTS}

\section{Overview findings}

Out of 50 patients in each arm, 41 underwent embryo transfer in traditional incubation and 45 in hypoxic incubation arm. Non transfers were mainly due to poor 
endometrial thickness, Risk of OHSS, difficulty encountered during transfer and patient wish. Overall OHSS incidence was $2 \%$. There were no incidence of empty follicle and fertilization failure. The pregnancy rate was $35 \%$ for hypoxic incubation as compared to $26 \%$ for incubation at atmospheric oxygen concentration (Table 1).

\section{Patient characteristic (age and BMI)}

Pregnancy rate was more for patient above age 45 years and in category 35-40 years, for rest the rate was comparable between the two incubation arms.

PR was significantly more for patients with BMI of 30 $\mathrm{kg} / \mathrm{m}^{2}$ in hypoxic incubation arm as compared to traditional incubation. Both for overweight and obese lady, hypoxic incubation gave better PR (Table 1 and 2).

\section{Embryology findings (fertilization and fragmentation rate)}

Fertilization rate for ICSI was markedly more in physiological incubation. The same is comparable for conventional IVF. In hypoxic incubation more ICSI embryos reached 4 cell stage. On further growth in hypoxic incubation more embryos reached 8 cell stage as compared to traditional incubation. This was seen for both ICSI and conventional IVF. For embryos left after transfer and vitrification, $68 \%$ and $73 \%$ for IVF and ICSI respectively reached blastocyst stage in hypoxic incubation arm.

Fragmentation rate as reflection of embryo quality was comparable for day 2 embryos in both traditional and hypoxic incubation conditions.

For 8 cell stage 163 of 197 embryos had minimal fragmentation. On further growth of these embryos $71 \%$ reached 4AA Gardners embryo grade category (Table 3 and 4).

\section{Embryology miscellaneous aspects (vitrification and day of transfer)}

More number of embryos were available for vitrification across all categories in hypoxic incubation. 16 patients in physiological incubation had D5 transfer as compared to 6 in traditional incubation (Table 5 and 6).

Table 1: Patient characteristics, age and PR.

\begin{tabular}{|lllll|}
\hline Age (years) & Number of patients & \multicolumn{2}{c|}{ Pregnancy rate } \\
\cline { 2 - 4 } & Traditional & Hypoxic & Traditional & Hypoxic \\
\hline $\mathbf{2 5}$ & 3 & 5 & 0 & 2 \\
\hline $\mathbf{3 0 - 3 5}$ & 9 & 11 & 3 & 3 \\
\hline $\mathbf{3 5 - 4 0}$ & 6 & 9 & 1 & 3 \\
\hline $\mathbf{4 0 - 4 5}$ & 11 & 8 & 3 & 3 \\
\hline$>\mathbf{4 5}$ & 7 & 5 & 3 & 2 \\
\hline Total & 5 & 7 & 1 & 3 \\
\hline
\end{tabular}

Table 2: Patient characteristics, BMI and PR.

\begin{tabular}{|lllll|}
\hline BMI & Number of patients & \multicolumn{3}{c|}{ Pregnancy rate } \\
\hline $\mathbf{1 1 8 . 5}$ & Traditional & Hypoxic & Traditional $(\%)$ & Hypoxic $(\%)$ \\
\hline $\mathbf{1 8 . 5 - 2 4 . 9}$ & 7 & 9 & $2(28)$ & $3(33)$ \\
\hline $\mathbf{2 5 - 2 9 . 9}$ & 11 & 13 & $5(45)$ & $4(30)$ \\
\hline$>$ 30 & 9 & 11 & $2(22)$ & $4(36)$ \\
\hline Total & 14 & 12 & $2(14)$ & $5(41)$ \\
\hline
\end{tabular}

Table 3: Embryology, overall growth characteristics.

\begin{tabular}{|c|c|c|c|c|c|c|c|c|}
\hline \multirow[b]{2}{*}{$\begin{array}{l}\text { Type of } \\
\text { incubation }\end{array}$} & \multicolumn{2}{|c|}{ Fertilization rate } & \multicolumn{2}{|l|}{4 cell stage } & \multicolumn{2}{|c|}{8 cell stage } & \multicolumn{2}{|c|}{ Blastulation rate } \\
\hline & IVF (\%) & $\begin{array}{l}\text { ICSI } \\
(\%)\end{array}$ & IVF $(\%)$ & $\begin{array}{l}\text { ICSI } \\
(\%)\end{array}$ & $\begin{array}{l}\text { IVF } \\
(\%)\end{array}$ & ICSI (\%) & $\begin{array}{l}\text { IVF } \\
(\%)\end{array}$ & ICSI $(\%)$ \\
\hline $\begin{array}{l}\text { Traditional } \\
\text { incubation }\end{array}$ & $221 / 291(76)$ & $\begin{array}{l}95 / 138 \\
(69)\end{array}$ & $179 / 221(81)$ & $\begin{array}{l}72 / 95 \\
(76)\end{array}$ & $\begin{array}{l}41 / 66 \\
(63)\end{array}$ & $12 / 23(56)$ & $\begin{array}{l}9 / 20 \\
(45)\end{array}$ & $1 / 5(20)$ \\
\hline $\begin{array}{l}\text { Hypoxic } \\
\text { incubation }\end{array}$ & $207 / 253(82)$ & $\begin{array}{l}198 / 21 \\
8(91)\end{array}$ & $173 / 207(84)$ & $\begin{array}{l}180 / 19 \\
8(91)\end{array}$ & $\begin{array}{l}93 / 113 \\
(83)\end{array}$ & $104 / 117(79)$ & $\begin{array}{l}31 / 45 \\
(68)\end{array}$ & $40 / 56(73)$ \\
\hline
\end{tabular}


Table 4: Embryology, fragmentation rate.

\begin{tabular}{|lllllll|}
\hline Type of incubation & \multicolumn{2}{l}{ Fragmentation rate 4 cell } & \multicolumn{2}{c|}{ Fragmentation rate 8 cell } & \multicolumn{2}{c|}{ Fragmentation rate blastocyst } \\
\cline { 2 - 7 } & Below 20\% & Above 20\% & Below 20\% & Above 20\% & Above 20\% & Above 20\% \\
\hline $\begin{array}{l}\text { Traditional } \\
\text { incubation }\end{array}$ & $178(71)$ & $72(29)$ & $32(61)$ & $20(39)$ & $4(40)$ & $6(60)$ \\
\hline Hypoxic incubation & $279(69)$ & $126(31)$ & $163(83)$ & $34(17)$ & $49(71)$ & $22(29)$ \\
\hline
\end{tabular}

Table 5: Vitrification.

\begin{tabular}{|llll|}
\hline Type of incubation & $\mathbf{4}$ cell & $\mathbf{8}$ cell & Blastocyst \\
\hline Traditional incubation & 91 & 8 & 2 \\
\hline Hypoxic incubation & 179 & 53 & 31 \\
\hline
\end{tabular}

Table 6: Transfer day.

\begin{tabular}{|lllllll|}
\hline Type of incubation & Day 2 $\operatorname{Tr}(\%)$ & PR (\%) & Day 3 $\operatorname{Tr}(\%)$ & PR (\%) & Day 5 Tr (\%) & PR (\%) \\
\hline $\begin{array}{l}\text { Traditional } \\
\text { incubation (41) 11 }\end{array}$ & $13(31)$ & $2(15)$ & $22(53)$ & $7(31)$ & $6(16)$ & $2(33)$ \\
\hline $\begin{array}{l}\text { Hypoxic incubation } \\
\text { (45) } 16\end{array}$ & $5(11)$ & $2(40)$ & $24(53)$ & $7(29)$ & $16(35)$ & $7(43)$ \\
\hline
\end{tabular}

\section{DISCUSSION}

The entire efforts since the very beginning of IVF-ET have been to provide an ideal environment to gametes to grow. It was aimed to mimic the same conditions as available inside the human body to maximize the efficiency of IVF program. This includes minimal and patient friendly stimulation. Air purification using high-efficiency particulate air (HEPA) filters to improve air quality. Reducing illumination intensity and exposure of embryos to light has also been proposed to reduce producing oxidative stress. ${ }^{3}$ Numerous animal studies using flexible microelectrodes introduced in genital tract have proven reduced oxygen tension as compared to environmental oxygen levels. ${ }^{4}$ This is also called physiological oxygen level. Various researchers have deduced this to be around $3-8 \%$ in humans.

Over time there has been improvement in media formulations. Additions of various essential and nonessential Amino acids have been made to meet the differing need of various stages of embryo growth. It was seen that following activation of embryonic genome at 8 cell, there is increased need of glucose for energy requirements. Addition of antioxidant combination of acetyl-L-carnitine, $\mathrm{N}$-acetyl-L-cysteine and $\alpha$-lipoic acid to culture media definitely improves the survival and robustness of embryos. ${ }^{5}$ Numerous endogenous antioxidants are richly present in follicular fluid to protect the cumulus oophorus complex from reactive oxygen species (ROS). These, namely melatonin also help in oocyte maturation, fertilization and embryo development. ${ }^{6}$

It was seen in numerous studies that tissue hypoxia under physiological conditions stimulates growth of various stem cells and also leads to increases pluoripotentency of mesenchymal cells. Early stages of embryonic growth are hastened by reduced oxygen tension. ${ }^{7}$ This was the basis of shifting to culturing embryos in reduced oxygen pressure. Increased production of ROS are blamed to cause decreased fertilization rate, reduced cleavage rate and reduced blastulation. Tissue hypoxia under physiological limits is postulated to reduce ROS. ${ }^{8}$ Reduced ROS leads to less oxidative damage to cell division cytoskeleton and improves mitochondrial functioning. We found 207 of 253 and 198 of 218 oocytes fertilized with IVF and ICSI respectively in hypoxic, physiological conditions of incubation as compared to a 221 of 291 embryos and 95 of 138 embryos in $20 \%$ oxygen environment. Hypoxic, $5 \%$ oxygen conditions gave us fertilization rate of $91 \%$ for ICSI embryos as compared to $69 \%$ in $20 \%$ oxygen. Age of both the partner has significant effect on IVF-ET cycle. Increase in age of female partner leads to reduced oocyte competence. With aging meiotic abnormalities accumulate over time. There may be disorder of meiotic spindle assembly. This leads to impaired chromosomal segregation, leading to aneuploidies. Similarly with aging of male partner competence of germ cell also reduces. ${ }^{9}$

In our study we saw pregnancy rate of female patient above the age of 45 years to be double in hypoxic incubation as compared to traditional incubation.

In our study of 100 patients we found an overall pregnancy rate of $36 \%$ for culturing embryos in 5\% oxygen using nitrogen gas to displace oxygen in eppendorf incubator as compared to $29 \%$ for culturing in ambient oxygen levels. HEPA filters, coda towers and air handling units were used to improve air quality in IVF lab. Antioxidant added culture media was used in all cases. Bontekoe et al in a Cochrane data base review found similar improved pregnancy rate and outcome of IVF cycles with 5\% oxygen levels in the incubator. In this meta-analysis of 1382 patients, they did not find any evidence of increased risk 
of multiple pregnancy, miscarriage rate or fetal abnormalities. This large systematic review and metaanalysis suggests that live birth can be improved by $30 \%$ in all ART clinics by culturing embryos in reduced oxygen pressure. ${ }^{10}$ Calzi et al studied 375 cycles in $20 \%$ oxygen and compared them with 306 low oxygen pressure cycles and found improved fertilization rate and quality of cleavage stage embryos. Because of increased ROS production in ambient $20 \%$ oxygen levels, it was felt to impair metabolomics of mitosis process of cleavage stage embryos. ${ }^{11}$ In conventional IVF definitive improvement in PR was found as compared to ICSI cycles. In our study we saw around $80 \%$ embryos reached day 3 in hypoxic $5 \%$ oxygen incubation condition as compared to about $60 \%$ in atmospheric oxygen for both IVF and ICSI. For day 2 transfer, we got a pregnancy rate of $40 \%$ for hypoxic incubation as compared to $15 \%$ for traditional incubation.

Dumoulin in a study of 1300 IVF cycles found improved blastulation rate in 5\% oxygen as compared to $20 \%$. Similar to our study, culture was done in micro droplet. It was hypothesized that micro droplet under oil produces a low oxygen microenvironment more suited for embryo growth. ${ }^{12}$ They found 236 of 310 embryos reached blastocyst stage in $5 \%$ oxygen environment as compared to 157 of 231 in $20 \%$ oxygen. In our study we found a blastulation rate of $68 \%$ and $73 \%$ for IVF and ICSI respectively for hypoxic incubation as compared to $45 \%$ and $20 \%$ respectively for traditional incubation.

Sobrinho et al found improved embryo growth with reduced oxygen tension. They found reduced pooled detrimental effect on embryo growth following prolonged incubation. ${ }^{13}$ This was especially pronounced on advanced embryo growth. In our study we found increased blastulation rate in $5 \%$ oxygen. PR at day 5 transfer was $43 \%$ in $5 \%$ oxygen as compared to $33 \%$ in $20 \%$ oxygen. In contrast to numerous studies we also found a significant improvement on PR for day 2 transfer for hypoxic incubation. Waldenström in a study of 396 infertile patients managed in low oxygen pressure using three gas system of $\mathrm{CO}_{2}, \mathrm{O}_{2}$ and nitrogen compared to two gas system of $\mathrm{CO}_{2}$ and environmental $20 \% \mathrm{O}_{2}$ also found more number of better quality embryos available for transfer. Improved day 5 growth in three gas system might be because of reduced cumulative damage by cytotoxic ROS generated due to prolonged culture and incubation. ${ }^{14}$ Similarly our study also showed improved fertilization rate especially for ICSI embryos. A rate of $91 \%$ as compared to $69 \%$ for traditional incubation. Day 5 growth was $73 \%$ as compared to $20 \%$ for traditional incubation.

Montfoort et al in a study of 871 patients for IVF found larger number of good quality embryos for cryopreservation when cultured in reduced oxygen pressure. ${ }^{15}$ The birth weight and outcome of frozen embryo transfer cycles were similar as compared to fresh transfers. We also found more number of good quality embryos available for vitrification. This will translate to more chances of pregnancy for our clientele as compared to culture in $20 \%$ oxygen. Our study found a significant improvement in embryo quality in 5\% oxygen as compared to $20 \%$ oxygen. This was more pronounced in day 3 and 5 embryos. We found $80 \%$ and $70 \%$ good quality embryos for day 3 and 5 as compared to 60 and 40 $\%$ for $20 \%$ oxygen respectively. Good quality embryos in our study were defined as embryos with less than $20 \%$ fragmentation, absence of multinucleation and equal blastomere.

Embryo quality may be evidenced by fragmentation rate as well functional competence. Ability to implant is reflected in PR. Physiological level of oxygen around 5\% leads to more competent embryos. Our study gave a PR of about $40 \%$ for day 2 and 5 embryos cultured in 5\% oxygen level.

Belli et al in an elaborate assessment of organelles by transmission electron microscopy (TEM) found that mitochondrial embryos cultured in physiological and environment oxygen levels are different. In early cleavage stage embryos, mitochondria's are spherical with dense cytoplasm and are sparse in distribution In $20 \%$ oxygen due to damage by ROS, TEM showed hooded, deformed mitochondria and vacuolation, with reduced membrane potential. ${ }^{16}$ This study shows that physiologically cultured embryos are anatomically healthier in their energy processing and utilization. Mitochondrial dysfunction has also been blamed for poor sperm motility and fertilization failure. Melatonin supplementation is proven to rescue the penetration capacity of impaired male factor. ${ }^{17}$

Abad in a study of more than 500 day 3 transfer assessed obstetric outcome of embryos cultured in low oxygen pressure. They found similar single and twin delivery rates; preterm delivery and very preterm delivery rates were similar. The mean birth weight, birth height, head circumference and 1 min APGAR scores were also similar between hypoxic and atmospheric oxygen titre groups. ${ }^{18}$ In our study for day 3, 8 cell embryos, we got $83 \%$ of good quality embryos defined as fragmentation rate below $20 \%$ and absence of multinucleation. Out of these 197 embryos, 49 embryos $(71 \%)$ reached 4AA stage of blastocyst development. These supernumerary good quality embryos give patient more chances of frozen embryo transfers in future. Thus enhancing the cumulative PR in hypoxic incubation cycles.

Ruíz et al found a significantly improved cumulative pregnancy rate and live birth rate with incubation in bench top incubator. ${ }^{19}$ Our study also showed improved pregnancy rate especially for D2 and D5 embryos.

Bavister proposed that detrimental effect of high oxygen pressure due to ROS may not be always evident morphologically. Apparently good quality embryos may have subtle molecular deficiency which impairs there implantation potentials. The cumulative deleterious effect on advanced incubation may be evident as reduced cells in Inner cell mass at blastocyst stage. This study argues for 
adopting low oxygen system Inspite of being costly as standard for all human IVF incubation. ${ }^{20}$ Guo also recommended low oxygen incubation for all IVF. By reducing damage by ROS to cleavage stage embryos, there is augmentation of their developmental potential. ${ }^{21}$ This leads to more robust embryos and improved blastulation rate. Thus, leading to healthier pregnancy and optimal outcome of IVF cycles.

\section{Limitations}

Sample size could be more to make data and findings more significant and the outcome of cycles and study of vitrification program was beyond the scope of the study.

\section{CONCLUSION}

We conclude that hypoxic incubation is more physiological. This leads to a healthier cohort of embryos. They are more robust and competent because of less oxidative damage. The pregnancy rates are higher. As more embryos are available for vitrification, cumulative pregnancy rate for IVF-ET cycles are definitely more.

As advanced culture is more efficient and blastulation rates are much higher in hypoxic incubation as compared to incubation in atmospheric oxygen levels, we recommended this method for embryo culture in human IVF.

\section{ACKNOWLEDGMENTS}

Authors would like to thank all the nursing staff, PG residents, interns and supportive staffs. The authors are really grateful to all the participating ladies who consented to be part of the study.

Funding: No funding sources Conflict of interest: None declared

Ethical approval: The study was approved by the Institutional Ethics Committee

\section{REFERENCES}

1. Dow K. The men who made the breakthrough: How the British press represented Patrick Steptoe and Robert Edwards in 1978. Reprod Biomed Soc Online. 2017;4:59-67.

2. Fischer B, Bavister BD. Oxygen tension in the oviduct and uterus of rhesus monkeys, hamsters and rabbits. J Reprod Fertil. 1993;99(2):673-9.

3. Noda Y, Goto Y, Umaoka Y, Shiotani M, Nakayama T, Mori T. Culture of human embryos in alpha modification of Eagle's medium under low oxygen tension and low illumination. Fertil Steril. 1994;62(5):1022-7.

4. García-Martínez S, Sánchez Hurtado MA, Gutiérrez H, Sánchez Margallo FM, Romar R, Latorre R, Coy $\mathrm{P}$, López Albors O. Mimicking physiological O2 tension in the female reproductive tract improves assisted reproduction outcomes in pig. Mol Hum Reprod. 2018;24(5):260-70.

5. Truong TT, Gardner DK. Antioxidants increase blastocyst cryosurvival and viability postvitrification. Hum Reprod. 2020;35(1):12-23.

6. Tamura H, Jozaki M, Tanabe M, Shirafuta Y, Mihara Y, Shinagawa M, et al. Importance of Melatonin in Assisted Reproductive Technology and Ovarian Aging. Int J Mol Sci. 2020;21(3):1135.

7. Podkalicka P, Stępniewski J, Mucha O, Trojanowska NK, Dulak J, Łoboda A. Biomolecules. Hypoxia as a Driving Force of Pluripotent Stem Cell Reprogramming and Differentiation to Endothelial Cells. 2020;10(12):1614.

8. Bedaiwy MA, Falcone T, Mohamed MS, Aleem AA, Sharma RK, Worley SE, Thornton J, Agarwal A. Differential growth of human embryos in vitro: role of reactive oxygen species. Fertil Steril. 2004;82(3):593600.

9. Cimadomo D, Fabozzi G, Vaiarelli A, Ubaldi N, Ubaldi FM, Rienzi L. Impact of Maternal Age on Oocyte and Embryo Competence. Front Endocrinol (Lausanne). 2018;9:327.

10. Bontekoe S, Mantikou E, van Wely M, Seshadri S, Repping S, Mastenbroek S. Low oxygen concentrations for embryo culture in assisted reproductive technologies. Cochrane Database Syst Rev. 2012;(7):CD008950.

11. Calzi F. Exposure of embryos to oxygen at low concentration in a cleavage stage transfer program: reproductive outcomes in a time-series analysis. Clin Lab. 2012;58(9-10):997-1003.

12. Dumoulin JC, Meijers CJ, Bras M, Coonen E, Geraedts JP, Evers JL. Effect of oxygen concentration on human in-vitro fertilization and embryo culture. Hum Reprod. 1999;14(2):465-9.

13. Sobrinho DBG. IVF/ICSI outcomes after culture of human embryos at low oxygen tension: a metaanalysis. Reprod Biol Endocrinol. 2011;9:143.

14. Waldenström U, Engström AB, Hellberg D, Nilsson $\mathrm{S}$. Low-oxygen compared with high-oxygen atmosphere in blastocyst culture, a prospective randomized study. Fertil Steril. 2009;91(6):2461-5.

15. Montfoort APAV.Reduced oxygen concentration during human IVF culture improves embryo utilization and cumulative pregnancy rates per cycle. Hum Reprod Open. 2020;2020(1):036.

16. Belli M, Zhang L, Liu X, Donjacour A, Ruggeri E, Palmerini MG, Nottola SA, Macchiarelli G, Rinaudo P. Oxygen concentration alters mitochondrial structure and function in in vitro fertilized preimplantation mouse embryos. Hum Reprod. 2019;34(4):601-11.

17. Zhang XY, Xiong YM, Tan YJ, Wang L, Li R, Zhang $\mathrm{Y}$, et al. Melatonin rescues impaired penetration ability of human spermatozoa induced by mitochondrial dysfunction. Reproduction. 2019;158(5):465-75.

18. Abad MR. The influence of oxygen concentration during embryo culture on obstetric and neonatal 
outcomes: a secondary analysis of a randomized controlled trial. Hum Reprod. 2020;35(9):2017-25.

19. Ruíz M. Effect of Group Embryo Culture under LowOxygen Tension in Benchtop Incubators on Human Embryo Culture: Prospective, Randomized, Controlled Trial. Reprod Sci. 2020;27(7):1522-33.

20. Bavister B. Oxygen concentration and preimplantation development. Reprod Biomed. 2004;9(5):484-6.

21. Guo N, Li Y, Ai J, Gu L, Chen W, Liu Q. Two different concentrations of oxygen for culturing precompaction stage embryos on human embryo development competence: a prospective randomized sibling-oocyte study. Int $\mathbf{J}$ Clin Exp Pathol. 2014;7(9):6191-8.

22. Plessis SSD, Hagenaar K, Lampiao F. The in vitro effects of melatonin on human sperm function and its scavenging activities on NO and ROS. Andrologia. 2010;42(2):112-6.

Cite this article as: Patil D. Does culture condition of reduced oxygen pressure helps in embryo quality? A prospective randomized study. Int J Reprod Contracept Obstet Gynecol 2021;10:2239-45. 